\title{
Strip-loaded nano-photonics on horizontal slot waveguide
}

\author{
Ségolène Pélisset ${ }^{*}$ (D) and Matthieu Roussey
}

\begin{abstract}
Background: A strip-loaded slot waveguide is a waveguide platform allowing a large amount of degrees of freedom in terms of fabrication. Contrary to other waveguide types where the guiding layer has to be patterned, only the shape of the top layer, usually a polymer, dictates the response of this platform.

Methods: We use the Finite Difference Time Domain method to study the field distribution and the modal behavior of light inside such a waveguide when the loading strip is patterned.

Results: We present an overview of several photonic-crystal-based structures on a strip-loaded slot waveguide platform. This theoretical study shows how the fundamental mode confined in the horizontal slab slot waveguide interacts with the dielectric loading structures. By investigating the field confinement, one determines the importance of the longitudinal shape over the lateral shape of the loading structure. Through the examples of sidewall corrugation, row of cylindrical or elliptical holes, and random shapes, we show how such a platform can help integration of complex functions in waveguides.

Conclusions: Although the spectral features depend strongly on the period and fill factor of the Bragg gratings studied in our case, the material distribution plays a key-role in the mode behavior. This impacts directly on the applications of such a platform for further modulators and sensors, for instance.
\end{abstract}

Keywords: Optical waveguides, Nanophotonics, Bragg grating, Refractive index distribution

\section{Introduction}

The constraint of Integrated Optics in term of power consumption pushes the research in the direction of low-loss devices, small footprint elements, and high efficiency components. Such requirements are synonym of highly challenging fabrication methods aiming at near-zero roughness of the waveguide structures, high refractive index materials yielding tight bends and smart hybridization of active materials in the components leading to enhanced optical response of the devices to a stimulus.

Over the years, three types of waveguide-integrated components - in addition to their combination leading to new kind of structures, but based on the same principles - emerged: the low index contrast waveguides (LIC), the high index contrast waveguides (HIC), and the loaded structures on HIC.

The first category consists in creating a higher refractive index region inside a bulk material. It includes ion

\footnotetext{
*Correspondence: segolene.pelisset@uef.fi

Institute of Photonics, University of Eastern Finland, 80101 Joensuu, Finland
}

exchange glasses devices [1, 2] and proton exchange components in lithium niobate or similar material $[3,4]$, for instance. They provide extremely low propagation losses values, but the small difference of refractive index between the core and the cladding of the waveguide does not allow sharp bends necessary for small footprint. The second type arose with silicon photonics [5], which enabled, in optics, the use of CMOS-compatible processes in a very high refractive index material at the telecom wavelengths $(1525 \mathrm{~nm}<\lambda<1575 \mathrm{~nm})$. The extreme footprint attainable in terms of bends for example in such components is as small as a few microns $[6,7]$. The third structure type originates naturally from the need of very thin bandwidth in HIC components $[8,9]$. It consists in a HIC waveguide on top or on the side of which a shallow pattern is created. It is indeed well known that a Bragg grating, for instance, can open a photonic band gap in the transmission spectrum of the waveguide in which it is engraved and the width of this band gap depends directly on the refractive index 
difference $(\Delta n)$ of the features [10]. Sidewall and top corrugation, i.e., shallow patterning of the side or top of the waveguide with amplitudes of a few tens of nanometers, were then used [11]. These features, affecting less the optical mode than a trench in the waveguide, provide narrower bandwidth, and are thus more adapted to wavelength (de)multiplexing.

Although these solutions provide exceptional results in terms of feature size, optical bandwidth, and enhancement, drawbacks remain concerning the versatility of the devices coming from the choice of the materials and the challenges implied by the fabrication techniques.

We propose in this article to investigate the possibilities offered by a fourth type of waveguide-based structure involving the integration of dielectric loading micro- and nanostructures on LIC waveguides. In our case, we consider a strip-loaded slot waveguide $[12,13]$ composed of two high refractive index rails separated by a few tens of nanometers thick low refractive index slab. By the slot waveguide effect [14], the electric field is confined in this thin slab while a polymer strip, patterned on top of this multilayered structure, confines the mode laterally. In such a platform, the fundamental quasi-TM mode is buried and only the end of the evanescent tail of the mode sees the loading strip and the structures patterned in it. It yields a minimization of the scattering losses arising from abrupt variation of refractive index. It is to be noted that the dispersion of each material constituting the waveguide structures presented in this article will affect slightly the response of the device by changing the mode confinement and the effective index of the waveguide. However, in order to keep the discussion focused on the patterning of the loading strip and the shape of the feature, we make the approximation of constant refractive indices within the considered wavelength range.

In this paper, we study first the effect of the loading strip pattern on the modal distribution of the fundamental quasi-TM mode, in order to evaluate how much light is lost by structuring the loading strip. In a second part, we analyze the field confinement in the plane of the horizontal slot waveguide $(x, z)$ to give a comprehensive understanding on the lateral field confinement with different feature shape, i.e., rounded sidewall corrugations
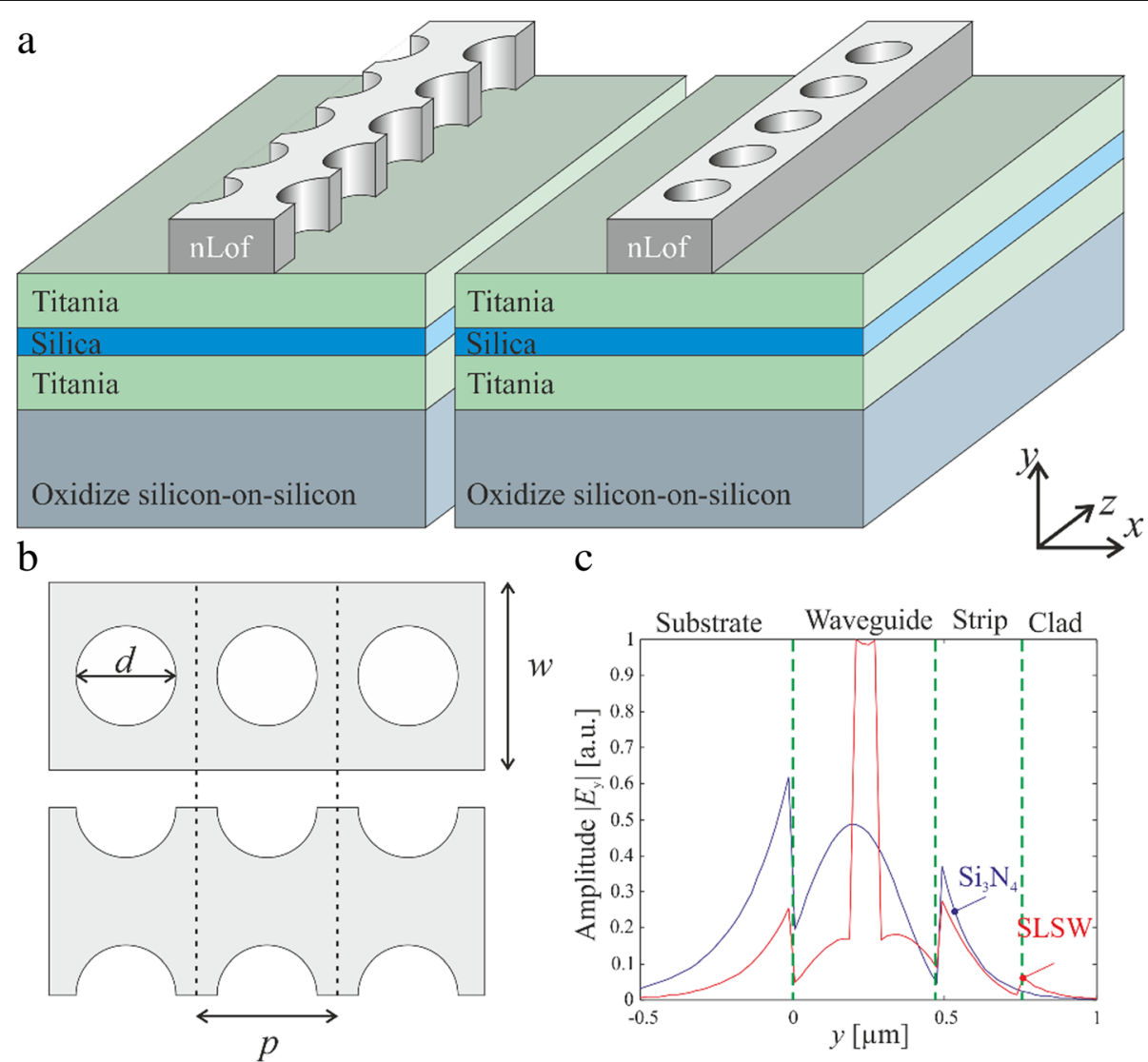

Fig. 1 a Sketch of the strip-loaded horizontal slot waveguide platform and the two main nanostructures studied in this article, i.e., rounded corrugations on the side wall of the polymer loading strip and a row of circular holes. b Top view and parameters of the two geometries. $\mathbf{c}$ Comparison of the quasi-TM fundamental mode profile $\left(E_{y}\right)$ in the case of a silicon nitride ridge waveguide (blue curve) and a strip-loaded slot waveguide (red curve) 
and circular holes. Finally, we open the discussion on the creation of arbitrary function using quasi-random two-dimensional patterning.

Figure $1 \mathrm{a}$ and $\mathrm{b}$ illustrate the basic structures we are studying in this article. It is composed of the horizontal slot waveguide with rail thickness $t_{\text {rail }}$, rail refractive index $n_{\text {rail }}$, slot thickness $t_{\text {slot }}$, and slot refractive index $n_{\text {slot }}<n_{\text {rail }}$. The lateral confinement is created by the polymer loading strip on top of the horizontal slot waveguide. It has a width $w_{\mathrm{S}}$ and a thickness $t_{\mathrm{S}}$. This polymer strip is considered periodically patterned. We define the period $p$, and the surface fill factor $f=\frac{S}{p^{2}}$, with $S$, the surface of the feature. It is important to note that $f$ is defined with regards to the surface of the period and not to the surface $p \times w_{\mathrm{S}}$ considering the width of the waveguide. This choice is made to keep independent the width of the waveguide and the shape of the feature.

\section{Results and discussion}

\section{Vertical mode confinement}

A strip-loaded horizontal slot waveguide confines the quasi-TM mode (electric field oriented along the

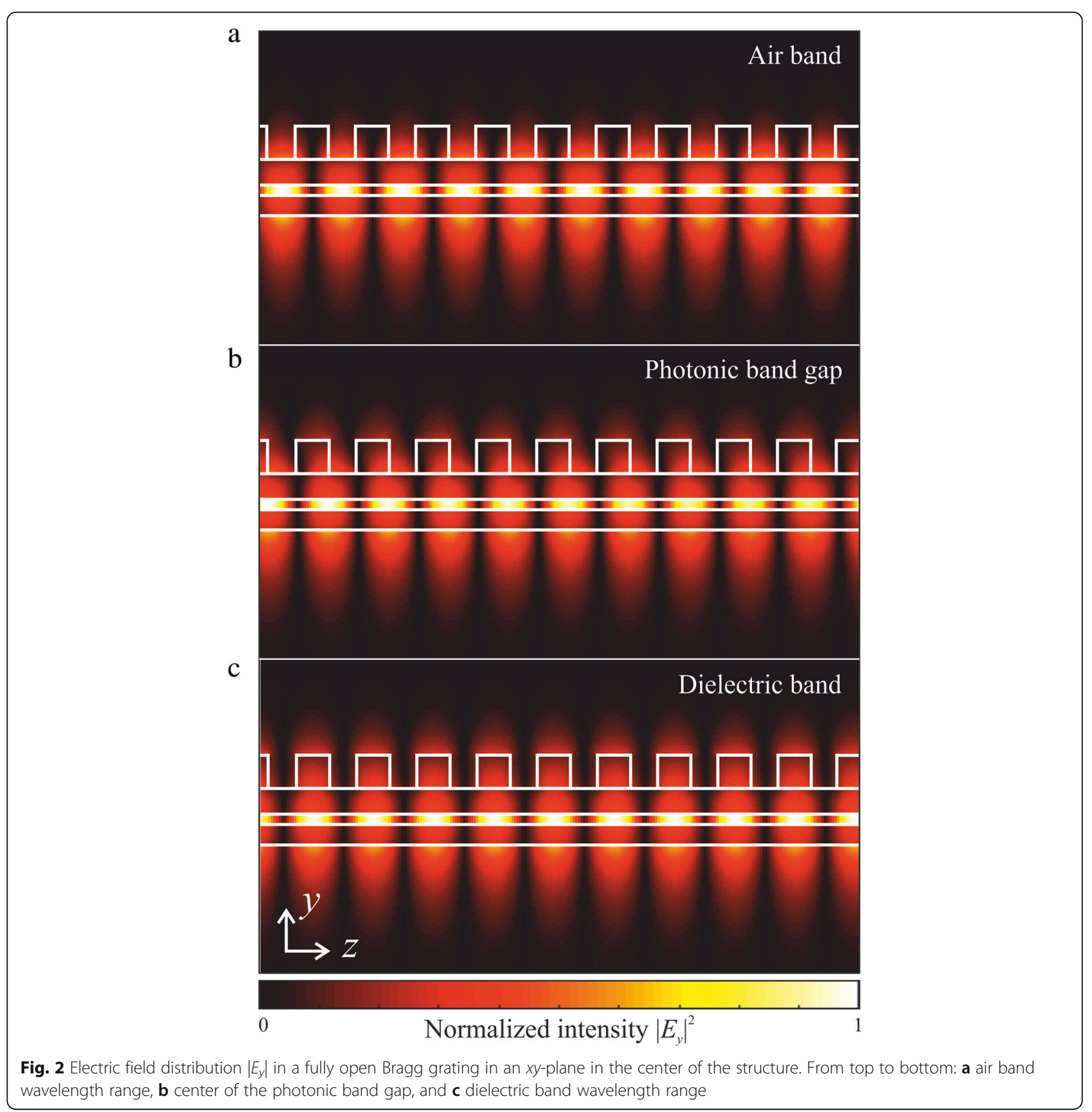


$y$-direction, see Fig. 1c) in the low refractive index region of the stack [12]. We consider here, $t_{\text {slot }}=80 \mathrm{~nm}, t_{\text {rail }}=$ $200 \mathrm{~nm}, t_{\mathrm{S}}=250 \mathrm{~nm}$, and $w_{\mathrm{S}}=1.2 \mu \mathrm{m}$, for the refractive index $n_{\text {slot }}=1.444\left(\mathrm{SiO}_{2}\right), n_{\text {rail }}=2.27\left(\mathrm{TiO}_{2}\right)$, and $n_{\mathrm{S}}=1.61$ (AZ nLOF) at the operating wavelength $\lambda=1550 \mathrm{~nm}$. The discontinuities at the boundaries lead to long evanescent tails existing in and even above the polymer strip. The advantage compared to a classical ridge waveguide is the part of the mode in interaction with the loading strip. This depends directly on the ratio of refractive index at the interfaces. This is illustrated in Fig. 1c), taking examples of the SLSW as described above, i.e., with polymer loading strip and $\mathrm{TiO}_{2}$ rails, and a silicon nitride $\left(n_{\mathrm{Si} 3 \mathrm{~N} 4}=1.9963\right)$ ridge waveguide of height $480 \mathrm{~nm}$ and width $1.2 \mu \mathrm{m}$. It is clear that in the case of the SLSW, most of the electric field is confined far under the surface, although the evanescent tail of the mode can still probe efficiently the surface where the polymer strip (and the nano-pattern) lies. All simulations in this article have been performed using the Finite Difference Time Domain method (OptiFDTD by OptiWAVE [15]) and the well-known transfer matrix method. After understanding the behavior of the electromagnetic field

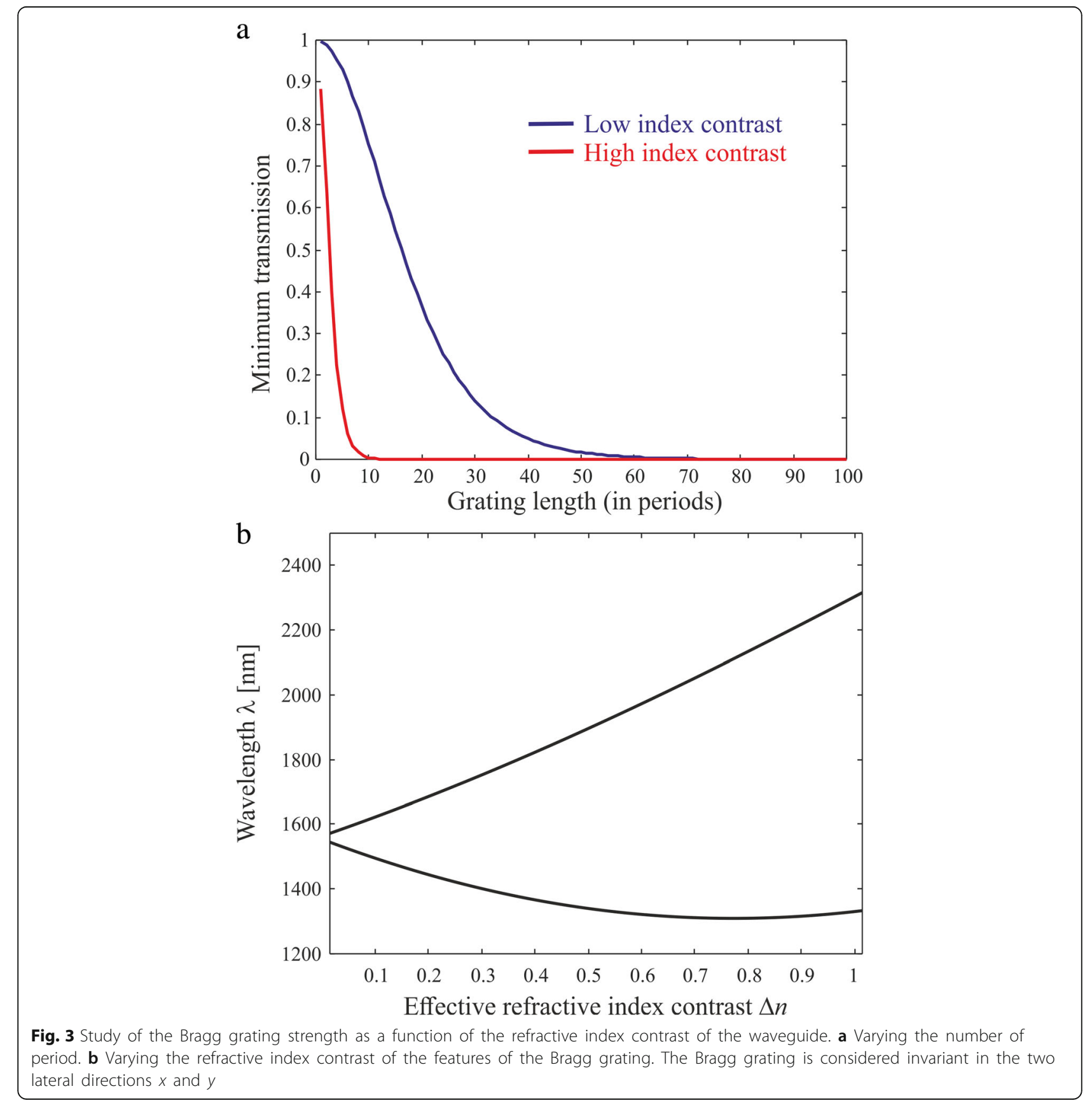


in the vertical direction, one can take advantage of working with LIC-waveguide-based structure, i.e., using the effective index approximation. This is not always valid for nanostructure on HIC waveguide [16], but very efficient in our case. This means that knowing the effective index of the fundamental mode of the SLSW with and without polymer (obtained from a previous study [12]), one can performed two-dimensional calculations.

To illustrate this effect we consider the field distribution of $\left|E_{y}\right|$ along a few periods in a $y z$-plane in the center of the structure. Results are presented in Fig. 2. A Bragg grating fully open (the pattern extended in the entire lateral direction of the loading strip) is considered in this case. A photonic band gap is opened and we observe the field at the different key-wavelengths of the spectrum, i.e., the air band edge (shorter wavelength), the dielectric band edge (longer wavelength), and the center of the photonic band gap. One can see that the field remains highly confined in the slot waveguide independently on the wavelength. One remarks also that the effect of the loading strip extends to the deeper layers of the stack creating the platform. The behavior of the field is classical for a photonic crystal structure with a higher confinement under the feature (holes, no material) for the air band and under the polymer material areas for the dielectric band. Finally, it is clear that no field is extensively leaking in the cladding layer and scattered out.

By monitoring the input and output field after a 400-period Bragg grating (fully open case), we have estimated the losses of the structure, by simulation, to 0.06 $\mathrm{dB} / \mu \mathrm{m}$ in the structure. Most of the field is leaking in the substrate.

\section{Effect of the lateral patterning}

In this section we study the effect of the patterning. Changing the refractive index of the loading strip by

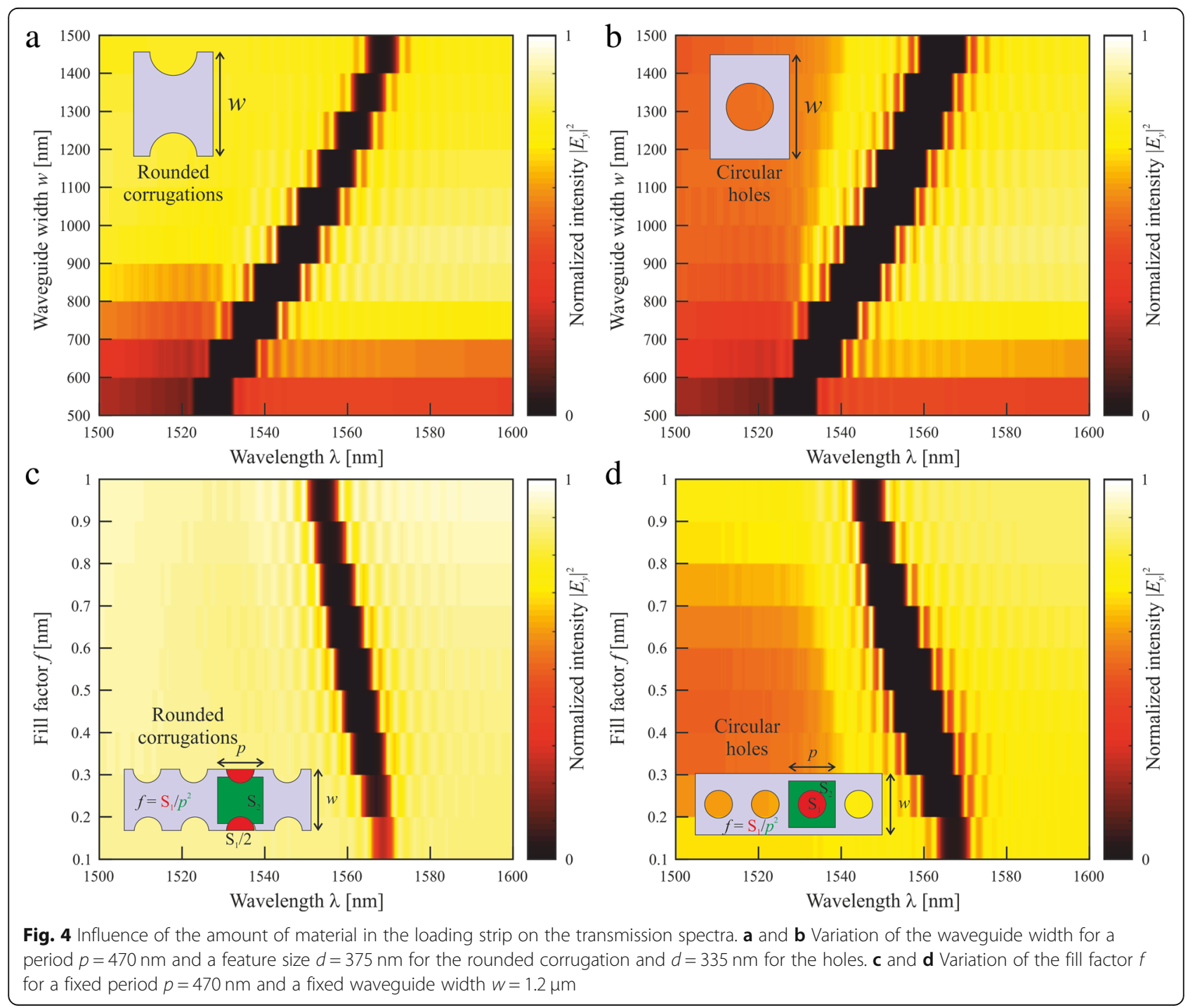


patterning it as a weaker influence than a direct pattern of the waveguide itself. It yields the necessity of longer rows of features in the propagation direction to obtain a significant opening of the photonic band gap, as shown in Fig. 3a). However, it allows a much thinner bandwidth of the photonic band gap, see Fig. 3b). The mode is buried and its overlap with the pattern is less than in the case of a strip waveguide, for instance. We showed in Ref. [12] that the width of the waveguide affects the shape of the mode, which changes from a Lorentzian shape to a Gaussian shape with an increasing width. Figure $4 \mathrm{a}$ and $\mathrm{b}$ show the effect of the waveguide width on the transmission spectrum of the structure for the two geometries. The first observation is a drift of the photonic band gap towards the longer wavelengths. One can also see that the intensity of the transmitted field is higher around the photonic band gap when the waveguide width is increased, i.e., the mode is Gaussian-like. It corresponds to less field outside the region under the loading strip, which means a better confinement and thus a higher interaction with the pattern. It also means less leaking in the slab-slot waveguide, as we will see later in this article. Figures $4 \mathrm{c}$ and $\mathrm{d}$ are illustrating the effect of the fill factor on the spectrum for the rounded
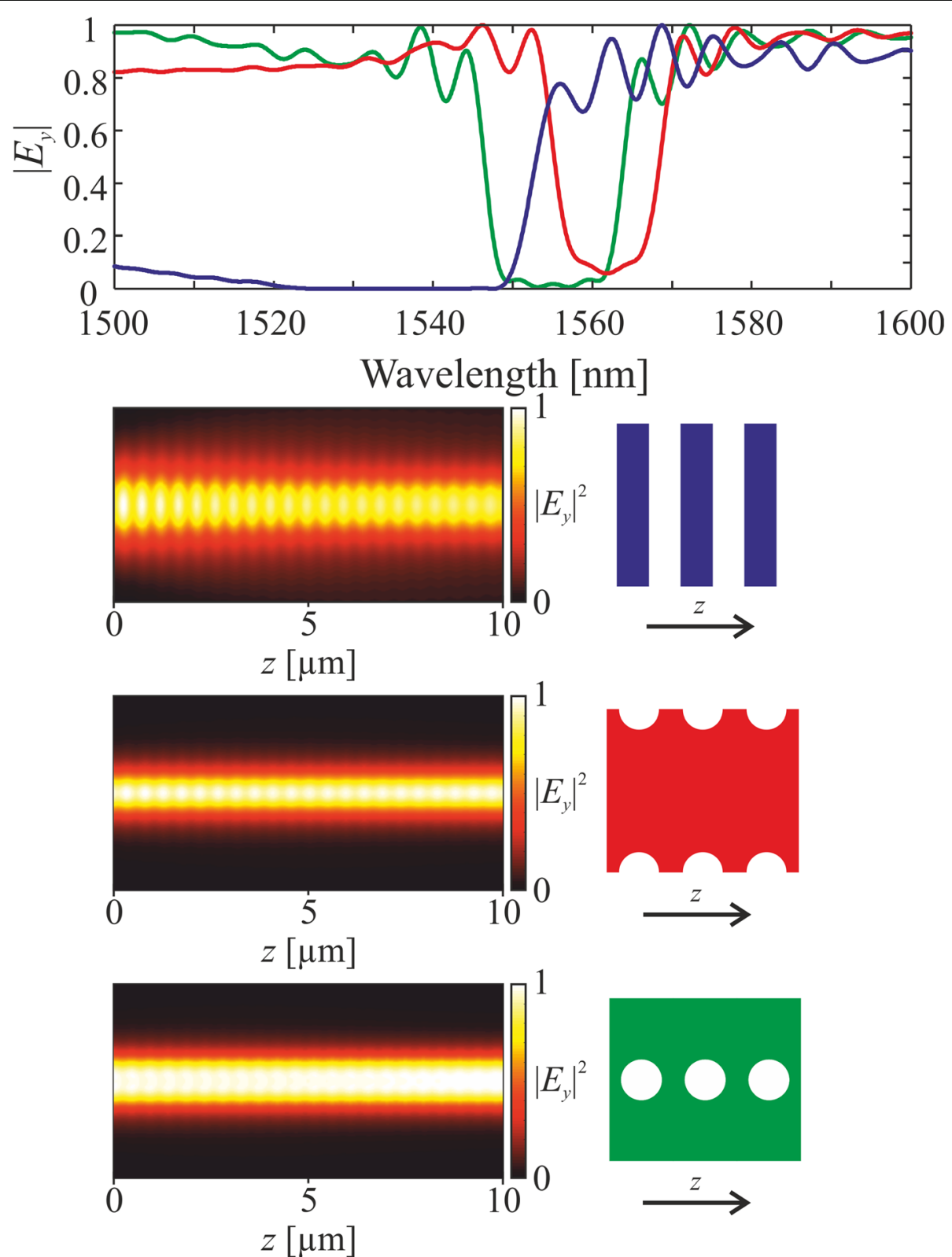

Fig. 5 Comparison of the spectra and in-plane mode distributions in the case of (top to bottom) a fully open Bragg grating (blue), a rounded corrugated sidewall grating (red), and a row of circular holes (green) in the center of the waveguide. The period is kept constant, $p=470 \mathrm{~nm}$ 
corrugation grating and the row of circular holes. It is to be noted once again that the fill factor is defined with respect to the period surface $\left(p^{2}\right)$ and is not related to the width of the waveguide but only to the feature itself. This explains why a photonic band gap exist also for extreme values of $f$. Such a choice allows us to understand how the material distribution on top of the horizontal slot waveguide affects the mode. One can see in both cases a slight shift of the photonic bandgap and small variation of its bandwidth. These results make clear that using a loading strip on top of a slot waveguide in a low index contrast waveguide configuration, i.e., no etching of the guiding layers, allows more relaxed fabrication. The structure is indeed less sensitive to small variations. One can see here also that the photonic band gap is more emphasized in the case of the rounded corrugation.

It becomes thus important to observe the field distribution of the mode inside the slot waveguide. Figure 5 displays the spectra and the $\left|E_{y}\right|$ distributions for a fully open Bragg grating, a rounded sidewall corrugation, and a row of circular holes, for a wavelength at the center of the photonic band gap. One can immediately see that the field tends to leak out of the waveguide (laterally) in the case of the simple Bragg grating while it remains centered under the strip in the two other cases. This is proven by the decrease of transmission at shorter wavelengths. Comparing the holes and the rounded corrugation, one can remark a more intense lateral confinement under the loading strip for the corrugated case. This proves that the field distribution is very sensitive to the material distribution although the amount of material remains the same.

\section{Towards random nanophotonics}

This brings us to consider different shapes for the feature while keeping the period and the fill factor constant. To simplify the interpretation of the results, we first consider a row of elliptical holes instead of circular ones. The top first spectrum in Fig. 6, shows how the photonic band gap opens. The extinction ratio is high, but the air band transmission is low. This is to be expected since this case of an ellipse elongated in the lateral direction, with respect to the waveguide propagation direction, is close to the fully open Bragg grating. Rotating the feature by an angle $\theta$ (from the $x$-axis) we progressively decrease the overlap between the mode and the feature, which is equivalent to a decrease of refractive index contrast. This leads to a reduced bandwidth and a lower extinction ration, while keeping the number of period constant to $N_{p}=200$, as we can see in Fig. 6. One can remark that an increasing $\theta$ goes with an increasing transmission on the edges of the photonic band gap. The side modulation is also considerably reduced. The

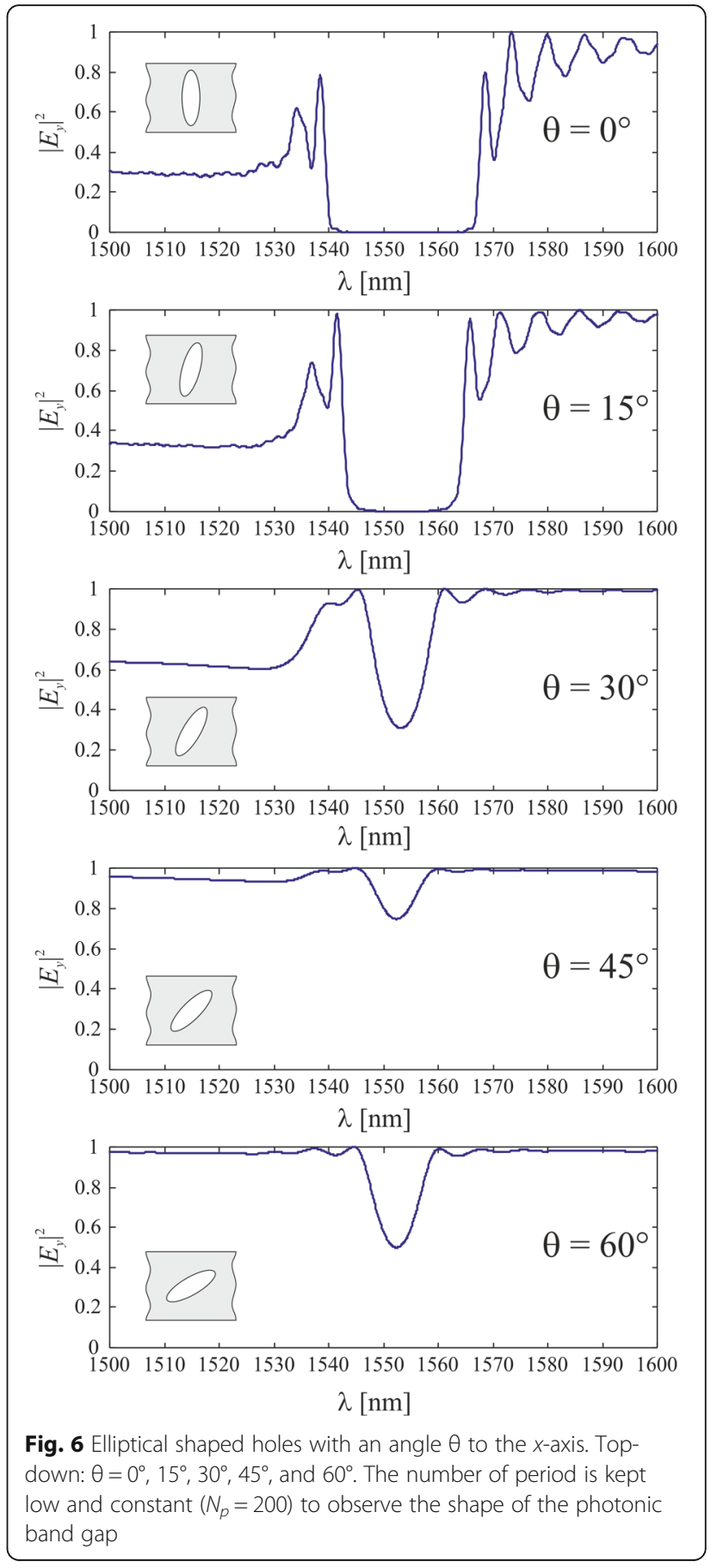

amount of material on the side of the features increasing, the field remains more confined under the strip. The $60^{\circ}$-case is particularly interesting since it corresponds to ellipses overlapping with the next period. It yields to an effective change of fill factor and results in a slightly higher extinction ratio.

To show that the material distribution does not affect much the spectrum in terms of position of photonic band gap, we performed simulation of an arbitrary shape 


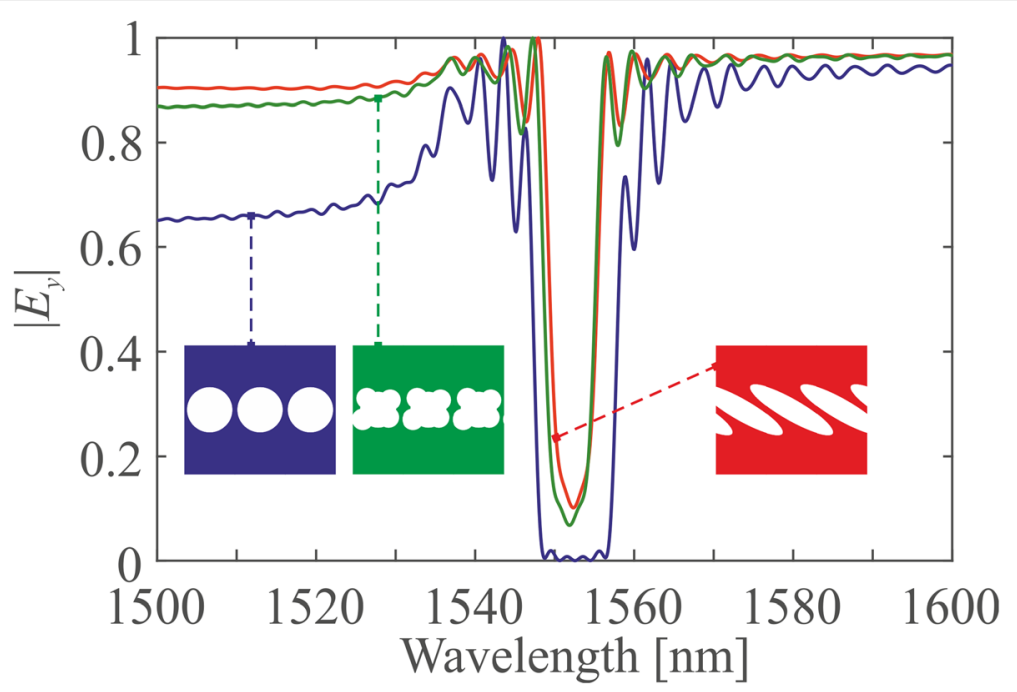

Fig. 7 Spectral response of a row of circular holes (blue), elliptic holes (red), and random holes (green). The period is kept to $p=470 \mathrm{~nm}$ and the fill-factor around $f=0.65$ to keep the central wavelength to $\lambda=1550 \mathrm{~nm}$

for the feature. The transmission spectra for this particular geometry, the row of holes and the $60^{\circ}$-tilted ellipses are shown in Fig. 7. One can see that only a change in the extinction ratio is relevant to mention. This allows us to conclude that the position of the photonic band gaps depends (beside the refractive index contrast) on $f$ and $p$. The material distribution affects the shape of the photonic band gap.

\section{Conclusion}

In this paper, we have demonstrated by simulation that nano-patterns on top of an SLSW platform can be realized with less demanding fabrication processes. The degree of liberty offered by this platform becomes of utmost importance when considering optics at different scale from millimeter to nanometer sizes.

Considering nanometric patterns, it is obvious that the interaction will be nearly negligible in the lateral direction while the effect will be significant in the propagation direction. One direction serves for spectral shaping while the other can be used as mode shaper, which is useful for loss reduction in bends, for beam splitting, coupling zones, and other (de)-multiplexers.

\section{Methods}

\section{Design and materials}

The SLSW platform has been conceived to operate at $\lambda=$ $1550 \mathrm{~nm}$. The two high refractive index rails are composed of titanium dioxide $\left(\mathrm{TiO}_{2}, n_{\text {rail }}=2.27\right)$ and have a thickness of $t_{\text {rail }}=200 \mathrm{~nm}$. The slot region is made of silicon dioxide $\left(\mathrm{SiO}_{2}, n_{\text {slot }}=1.44\right)$ for a thickness $t_{\text {slot }}=80$ $\mathrm{nm}$. The layers have been deposited on an oxidized silicon wafer by atomic layer deposition [12]. The thermal oxide layer has a thickness of $3 \mu \mathrm{m}$. The pattern (or strip) on top of the stack is a resist for electron beam lithography (AZ-nLof - 2070, MicroChemicals) with refractive index $n_{\mathrm{s}}=1.6$.

\section{Effective index}

To simplify the simulations we used the effective index approximation method. It allows to reduce to two dimensions a three-dimensional problem. The effective indices of the fundamental TM mode (slot mode) with and without the loading polymer layer are 1.7081 And 1.6171 , respectively.

\section{Finite difference time domain method}

The finite difference time domain (FDTD) method is based on the discretization in space and time of the Maxwell's equations [17]. It allows a computation of the electromagnetic field inside the structure and a visualization of the modes considering TM-polarized light $\left(E_{y}\right)$. We performed two-dimensional simulations (OptiFDTD by OptiWAVE [15]). In the case of Fig. 2, we observed the field propagating in a structure invariant in the $x$-direction. We used for these calculations the refractive indices of the materials (measurements done by ellipsometry). In all other cases, we used the effective index approximation as described above. This allows to take into account the vertical confinement of the mode while reducing the calculation time.

\section{Abbreviations}

HI: High index; LI: Low index; SLSW: Strip-loaded slot waveguide

Acknowledgements

Not applicable. 
Funding

We acknowledge funding from the Business Finland under the project ChemiReader (No 2387/31/2017).

\section{Availability of data and materials}

The datasets supporting the conclusions of this article are included within the article.

\section{Authors' contributions}

SP performed the simulations. MR supervised the work. SP and MR wrote the manuscript. Both authors read and approved the final manuscript.

\section{Competing interests}

The authors declare that they have no competing interests.

\section{Publisher's Note}

Springer Nature remains neutral with regard to jurisdictional claims in published maps and institutional affiliations.

Received: 1 March 2019 Accepted: 22 April 2019

Published online: 06 May 2019

\section{References}

1. Tervonen, A., West, B.R., Honkanen, S.: lon-exchanged glass waveguide technology: a review. Opt. Eng. 50, 071107-071-15 (2011)

2. Araci, I.E., Himmelhuber, R., DeRose, C.T., Luo, J.D., Jen, A.K.-.Y., Norwood, R. A., Peyghambarian, N.: Alignment-free fabrication of a hybrid electro-optic polymer/ion-exchange glass coplanar modulator. Opt. Express. 18, 2103821046 (2010)

3. Bazzan, M., Sada, C.: Optical waveguides in lithium niobate: recent developments and applications. Appl. Phys. Rev. 2, 040603 (2015)

4. Roussey, M., Bernal, M.-P., Courjal, N., Salut, R., Van Labeke, D., Baida, F...: Electro-optic effect exaltation on lithium niobate photonic crystals due to slow photons. App. Phys. Lett. 89, 241110 (2006)

5. Lipson, M.: Guiding, modulating, and emitting light on silicon - challenges and opportunities. J. Light. Technol. 23, 4222-4238 (2005)

6. Bogaerts, W. De Heyn, P. Van Vaerenbergh, T., De Vos, K., Selvaraja, S.K. Claes, T., Dumon, P., Bienstman, P., Van Thourhout, D., Baets, R.: Silicon microring resonators. Laser Photonics Rev. 6, 47-73 (2012)

7. Cherchi, M., Ylinen, S., Harjanne, M., Kapulainen, M., Aalto, T.: Dramatic size reduction of waveguide bends on a micron-scale silicon photonic platform. Opt. Express. 21, 17814-17823 (2013)

8. Wang, X., Shi, W., Yun, H., Grist, S., Jaeger, N.A.F., Chrostowski, L.: Narrowband waveguide Bragg gratings on SOI wafers with CMOS-compatible fabrication process. Opt. Express. 20, 15547-15558 (2005)

9. Furuta, H., Noda, H., Ihaya, A.: Novel optical waveguide for integrated optics, Appl. Opt. 13, 322-326 (1974)

10. Joannopoulos, J.D., Johnson, S.G., Winn, J.N., Meade, R.D.: Photonic Crystals: Molding the Flow of Light - Second Edition. Princeton University Press, Princeton, New Jersey (2008)

11. Paul, S., Roussey, M., Kuittinen, M., Honkanen, S.: Multi-wavelength add-drop filter with phase-modulated shifted Bragg grating. Opt. Lett. 43, 3144-3147 (2018)

12. Pélisset, S., Laukkanen, J., Kuittinen, M., Honkanen, S., Roussey, M.: Modal properties of a strip-loaded horizontal slot waveguide. J. Eur. Opt. Soc.-R.P. 13 (2017)

13. Roussey, M., Ahmadi, L., Pélisset, S., Häyrinen, M., Bera, A., Kontturi, V., Laukkanen, J., Vartiainen, I., Honkanen, S., Kuittinen, M.: Strip-loaded horizontal slot waveguide. Opt. Lett. 42, 211-214 (2017)

14. Almeida, V.R., Xu, Q., Barrios, C.A., Lipson, M.: Guiding and confining light in void nanostructure. Opt. Lett. 29, 1209-1211 (2004)

15. https://optiwave.com/ (2/05/2019)

16. Qiu, M.: Effective index method for heterostructure-slab-waveguide-based two-dimensional photonic crystals. Appl. Phys. Lett. 81, 1163-1165 (2002)

17. Taflove, A., Hagness, S.C.: Computation Electrodynamics: the FiniteDifference Time Domain Method. Artech House, Norwood (2005)

\section{Submit your manuscript to a SpringerOpen ${ }^{\circ}$ journal and benefit from:}

- Convenient online submission

- Rigorous peer review

- Open access: articles freely available online

- High visibility within the field

- Retaining the copyright to your article

Submit your next manuscript at $\boldsymbol{\nabla}$ springeropen.com 